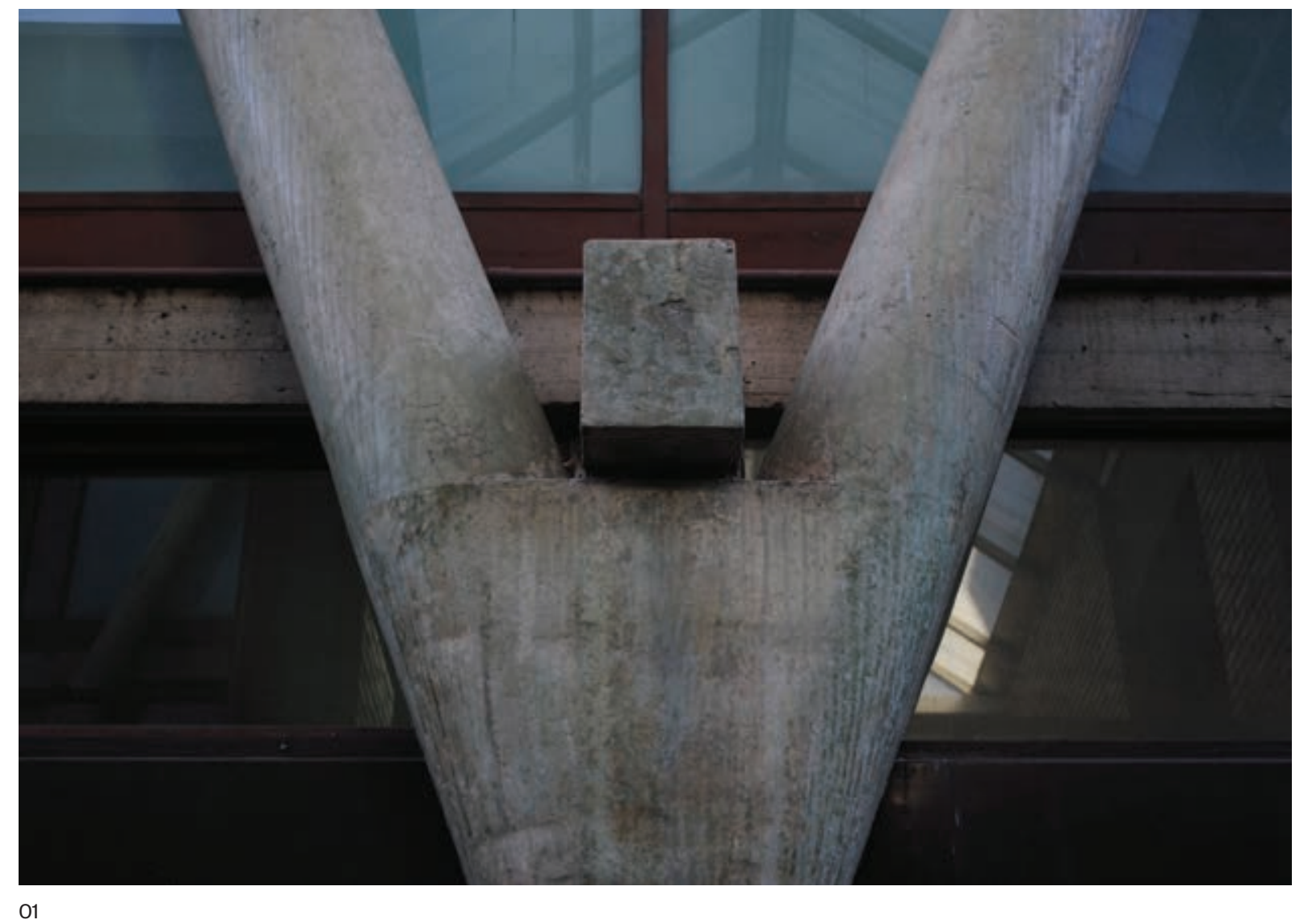




\section{Cualidad material en la arquitectura orgánica. El edificio para la Facultad de Arquitectura de Mendoza de Enrico Tedeschi Silvia Alvite}

Junto al florecimiento teórico de una tendencia arquitectónica organicista promovida desde mediados de la década de 1940 por un grupo de arquitectos italianos en torno al concepto del espacio, una paralela línea de investigación desarrolló el camino de las bioestructuras que conformaban sistemas de sostén integrados a la envolvente. En Argentina ambas vertientes tuvieron canales de experimentación durante la década de 1950 y un caso que condensa la madurez de aquellas propuestas de un modo singular es el edificio de la Facultad de Arquitectura de Mendoza proyectado por el arquitecto italiano Enrico Tedeschi entre 1960 y 1964. El proyecto explora sistematicidad, racionalidad constructiva y organicidad, rechazando la neutralidad de la materia en favor de la exploración de un campo táctil en el cual los límites del espacio se perciben desdibujados por un juego de sombras, transparencias y efectos dinámicos que acompañan una sutil mímesis naturalista.

\section{PALABRAS CLAVE}

Enrico Tedeschi, arquitectura orgánica, materia, naturaleza, estructura

\section{KEYWORDS}

Enrico Tedeschi, Organic Architecture, Material, Nature, Structure

FORMA,

ESPACIOY

MATERIA EN LA

ARQUITECTURA

ORGÁNICA

En la teoría y práctica arquitectónica las analogías naturalistas fueron fuente de inspiración desde siempre y, según Waenerberg, se aplicaron tanto a imitaciones figurativas como a formas estructurales o a estrategias compositivas'. Una de ellas fue la que difundió el arquitecto y crítico romano Buno Zevi a mediados de los años cuarenta en Italia. Su teoría sobre la "arquitectura orgánica" rechazaba la definición formal regulada mediante proporciones clásicas, la composición volumétrica y cualquier tipo de estandarización sosteniendo, en cambio, como valor prioritario, la definición del espacio interior como génesis de la forma exterior. Se trataba de una posición que, como

\section{Silvia Alvite}

Nació en Buenos Aires en 1977. Es arquitecta por la Universidad de Buenos Aires, Magíster en Teoría y Práctica del Proyecto de Arquitectura por la Universidad Politécnica de Cataluña y Doctora en Arquitectura por la Universidad de Buenos Aires. Actualmente es Profesora Adjunta de Teoría de la Arquitectura l y ll en la Universidad Nacional de San Martín, Argentina. Su tesis doctoral estudia la evolución de las ideas en el pensamiento y obra del arquitecto italiano Enrico Tedeschi a la luz de los cambios transcurridos en la teoría y en la práctica de la disciplina durante la segunda mitad del siglo $X X$ en América Latina.

E-Mail: silvia.alvite@fadu.uba.ar

Fig. 01

Detalle de la unión entre las piezas cónicas, el nudo, la viga transversal y el forjado. Fotografía de la autora, 2011. 
explica Hvattum, buscó enfrentar la idea mecánica de la forma impuesta desde afuera con la idea de un proceso orgánico que autorregula la forma desde adentro ${ }^{2}$. La intención de la propuesta de Zevi era reemplazar las opciones estéticas de los regímenes totalitarios por otras que promovieran la individualidad, la libertad formal y el espacio como esencia de la arquitectura; para ello, el principal modelo a seguir era la obra del arquitecto norteamericano Frank Lloyd Wright ${ }^{3}$. Sin embargo, la idea de que el espacio constituye la materia prima de la arquitectura fue debatida entre los propios defensores del movimiento orgánico italiano. Samonà afirmaba que se trataba de una combinación de espacio y materia ya que la arquitectura de Wright no podía explicarse por la forma geométrica de las configuraciones espaciales internas sino que debía hacerse por la continuidad y la plasticidad de una "materia espacializada"4. Por su parte, Argan consideraba que para alcanzar la aplicación proyectual de las ideas de Wright era preciso comprender que su esfuerzo era el de sublimar la naturaleza en la arquitectura a través de un proceso por el cual la "materia toma forma" por medio de una reinterpretación atravesada por la geometría y las reglas de la razón 5 . Como advierte Pope, el pensamiento organicista en la arquitectura no busca la imitación visual de un elemento natural sino que convoca a una interacción entre el objeto arquitectónico y el entorno natural y construido a través de la imitación de las relaciones funcionales de la naturaleza ${ }^{6}$.

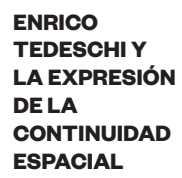

Luego de la segunda guerra mundial se desempeñó como urbanista en planes de reconstrucción para Italia e integró, junto a Zevi, Piccinato y otros arquitectos italianos, la Associazione per I'Architettura Organica (APAO) y el consejo directivo de la revista Metron. En 1948 emigró a Argentina y fue un destacado profesor en distintas universidades. Si bien se dedicó principalmente a la enseñanza fue un personaje multifacético dentro de la disciplinas de la arquitectura y el urbanismo, como profesional, teórico y técnico en planificación. Los mayores logros de su carrera académica los obtuvo como decano de la Facultad de Arquitectura de la Universidad de Mendoza entre 1961 y 1972. Luego, dedicó los últimos años de su vida a la investigación, optando por una aproximación ecológica a la arquitectura desde el desarrollo de tecnologías de energía solar aplicada al diseño de viviendas.

En los años cincuenta Tedeschi fue un portavoz de la arquitectura orgánica a través de sus programas pedagógicos y publicaciones en castellano. Promovió las mismas líneas espacialistas planteadas por Zevi, aunque sin los argumentos de tono político que no encajaban en el contexto de América Latina. Mediante un llamado a la liberación formal Tedeschi enfrentaba la abstracción figurativa como herramienta proyectual por asociarla a una homogeneización estandarizante y proclamaba la revalorización del factor humano y su singularidad para "[ ] superar la experiencia cubista, que valora sobre todo el volumen y la proporción"'. En 1955 dedicó un ensayo crítico a la figura de Frank Lloyd Wright, destacando en su obra el uso de los materiales en su expresión natural, la afinidad por el paisaje, la expresión formal libre y 


\section{ESTRUCTURA CONTEMPORANEA EN ARQUITECTURA ENRIOO TEDRSOHI, ARQ}
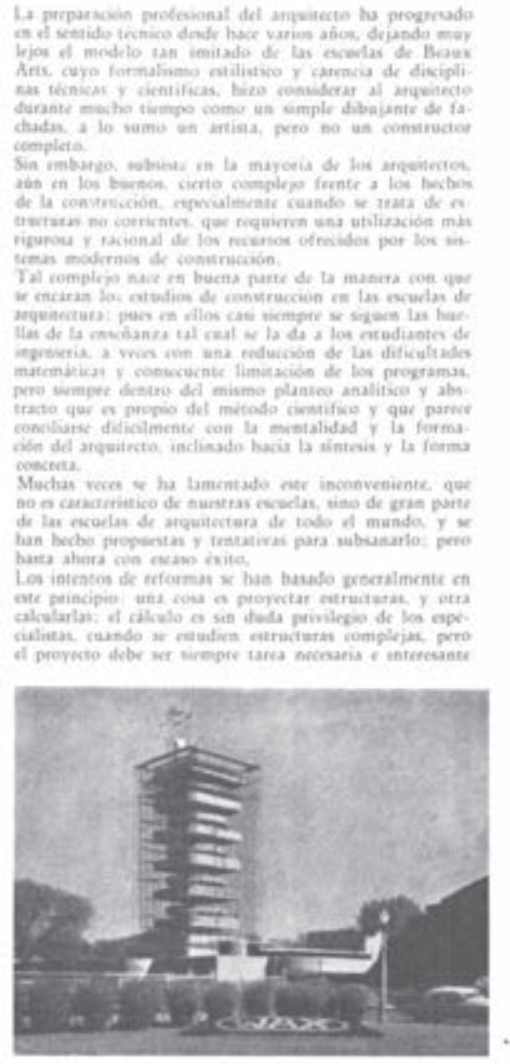

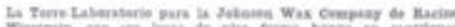

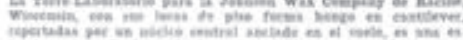

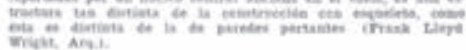

152

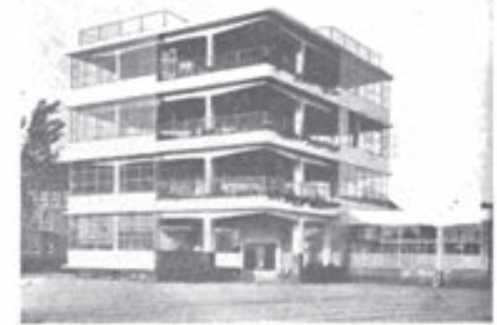

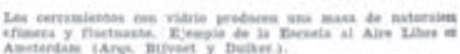

del arquiteoso. Conceptwalereir daro y simple on ajo

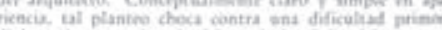

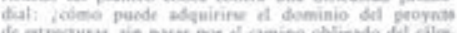

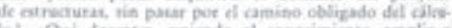
o' iou docrntes uniredo at conocimiento amplie o seguro det cblculo is schsihilidad aridord que les permidu acrorare a is mescalidad det ampeitecte penden ferjar an

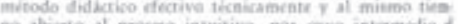

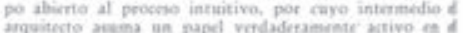
eqquitecto aweins an papel verdadefamente antivo et

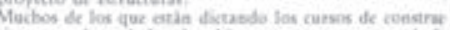
chition

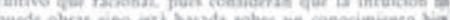

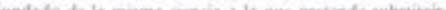

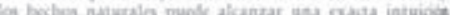

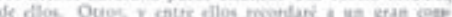

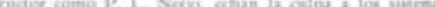

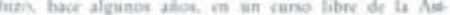

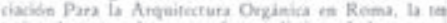

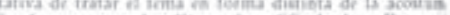

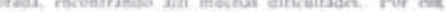

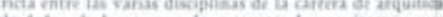

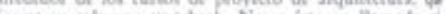

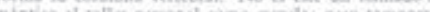

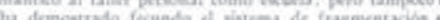

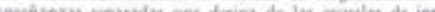

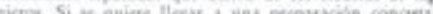

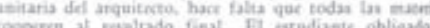

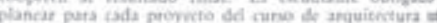

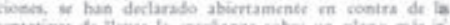

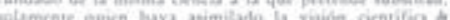

el rol del espacio en la generación plástica ${ }^{8}$. Algunos años más tarde fue profundizando este enfoque en complemento de aspectos marcados por un matiz ambientalista que lo fue acercando a un interés especial por las estructuras y el clima. Eran épocas en las que los factores psicológicos y perceptuales se habían introducido en los métodos del proyecto arquitectónico incorporando las variables de la visión en movimiento del espacio y la transparencia? ${ }^{9}$ Tedeschi no confiaba en que el progreso de la tecnología pudiera resolver los problemas estéticos aunque tampoco era partidario de una mirada artesanal ni vernácula hacia los materiales. Se interesaba por los avances industriales y apreciaba los ensayos con sistemas de prefabricación modular desarrollados en la segunda posguerra en Europa. El acierto de estos sistemas, según Tedeschi, estaba en que los elementos estándar, regulados por retículas, otorgaban flexibilidad, capacidad de crecimiento e interés estético, siempre y cuando no cayeran en la monotonía:

"Es el sistema del que tan fatigosamente tratamos de salir en estos últimos años para alcanzar expresiones de continuidad espacial y plástica más acercadas a nuestra visión dinámica del contorno"10. 
En ocasión de publicar un artículo sobre el diseño estructural en la arquitectura, Tedeschi ilustraba la búsqueda del valor estético y la creatividad en las estructuras con los casos del edificio Johnson Wax en Wisconsin de Wright y de la Escuela al Aire Libre en Amsterdam de los arqs. Bijvoet y Duiker, ejemplos en los que una estructura constitutiva de la envolvente -y apoyada por la disolución de la apariencia sólida del volumen-era la clave de la expresión global" (fig. 02). Su interés en la expresión estructural lo condujo a valorar los estudios de los ingenieros contemporáneos que articulaban conocimientos técnicos con principios formales provenientes del mundo biológico ${ }^{12}$. En su obra didáctica más difundida, Teoría de la arquitectura, Tedeschi hacía referencias a trabajos de Torroja, Nervi y D' Arcy Thompson y exponía argumentos a favor de la optimización de la forma para obtener la mejor performance estructural del material, aunque advertía que la aproximación proyectual de los ingenieros por lo general carecía de sensibilidad por la escala humana, aspecto que consideraba de sumo valor en la arquitectura ${ }^{13}$. No obstante, no se trataba para Tedeschi de un enfrentamiento entre disciplinas, ya que tampoco celebraba la búsqueda formal y espacial cuando estuviera deslindada de la eficiencia y naturaleza propia del material, caso que identificaba en las obras de Oscar Niemeyer en Brasilia, a las que oponía las estructuras de Pier Luigi Nervi en Turín:

\footnotetext{
"Observemos cómo la bóveda de Turín se transforma en sus apoyos en elegantes abanicos, de formas que recuerdan los tallos de las palmeras; la extraordinaria finura de los arcos vertebrados y de las piezas costillares que los unen, donde se refirma (sic) el sentido naturalista y orgánico de la estructura: la firme sensación de escala humana, fácil de perder en semejante dimensión estructural, producida por las galerías y balcones laterales; la ilumniación profusa que trasparenta el techo y le da vuelo. Nuestra admiración se multiplica”14.
}

El ingeniero Nervi fue una figura cercana a Tedeschi. Ambos habían formado parte de la Escuela de Arquitectura Orgánica en Roma entre 1946 y 1947 y, poco después, Nervi desarrolló el diseño y cálculo estructural de la cubierta para el Centro Comunal de la Ciudad Universitaria de Tucumán, Argentina (1948), proyecto en el cual Tedeschi participó (fig. 03). Giulio Pizzetti -otro ingeniero italiano que vivió algunos años en el país- también promovió desde su cátedra en la Universidad de Buenos Aires las metodologías de diseño estructural apoyadas en el ensayo empírico con modelos y colaboró, entre otras obras, con el arquitecto Amancio Williams en el proyecto experimental de bóvedas cáscara para los hospitales de Corrientes (1948-53) ${ }^{15}$. Por su parte, el arquitecto argentino Eduardo Catalano, partícipe del proyecto de Tucumán, emigró a los Estados Unidos en los años cincuenta y dedicó un importante período de investigación a los estudios estructurales en base a piezas modulares y superficies continuas y regladas (fig. 04). Las experiencias estructuralistas, aunque no fueron muchas, eran bien conocidas por Tedeschi.

EL EDIFICIO PARA
LA FACULTAD DE
ARQUITECTURA DE
LA UNIVERSIDAD
DE MENDOZA

dirección del proyecto para el edificio de su sede con la colaboración de los ingenieros Diego Franciosi en el diseño y montaje de los elementos 


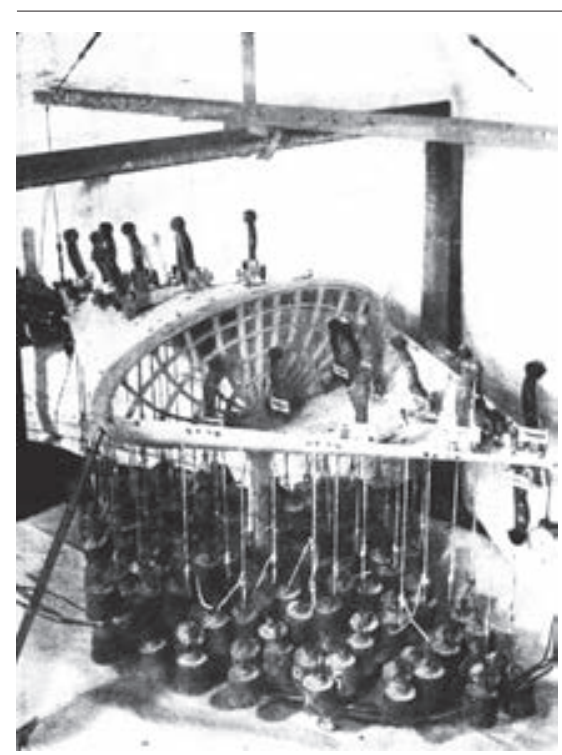

03

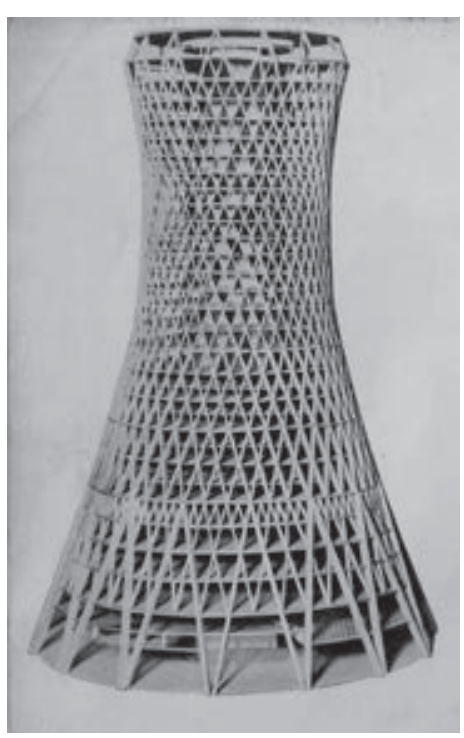

04

prefabricados y Roberto Azzoni en el cálculo generalir. El proyecto resuelve con efectividad una estructura antisísmica de gran elegancia alcanzando una racionalización material de un modo complejo, condición que se tradujo, como indica Codina, en una innovación técnica que no se apoya en los usos y conocimientos constructivos tradicionales del materialr ${ }^{r}$. Debido a una intención de trabajar con bajos costos en la construcción se utilizaron piezas prefabricadas producidas por la empresa SCAC, algunas destinadas a edificios -como las vigas y losetas premoldeadas de hormigón armado pretensado utilizadas en los entrepisos y la cubierta- y otras destinadas en su origen a postes de iluminación urbana que, en esta obra, materializan los segmentos de una retícula que configura los lados principales del volumen, al frente y contrafrente ${ }^{18}$. Estos últimos elementos, de hormigón centrifugado, se desarrollan en forma cónica debido a que su sección disminuye a medida que aumenta su altura; un dato esencial para la definición del proyecto, ya que se utilizaron como tramos de una retícula que decrece su sección en conjunto con el desarrollo vertical de la fachada del edificio. La disposición diagonal de la retícula obedece principalmente al objetivo de reducir las deformaciones ante la necesidad de resistencia al sismo. La disminución progresiva en la sección de las piezas cónicas, sumada al efecto dinámico de la dirección diagonal de la trama generan perceptivamente un movimiento ascendente, efecto que permite que una composición regular y de construcción mecánica, tenga una apariencia orgánica. Un momento clave del proceso, según el propio autor, fue cuando debió reflexionar acerca del diseño de los nudos entre los postes:

"[ ] si se resolvieran con una forma simple y continua los nudos de unión del reticulado diagonal de estos postes cónicos, ¿no podría lograrse ese carácter orgánico y natural que el ambiente del parque sugiere, presentando las columnas como elementos que nacen del suelo y se van haciendo más esbeltos al elevarse, así como hacen los troncos de los árboles?"19.

El diseño de los nudos y de los pies de apoyo disuelve la identificación de las partes y contribuye a la lectura en crecimiento de la

Fig. 03

Pier Luigi Nervi. Ensayos para la estructura de la cubierta del Centro Comunal de la Ciudad Universitaria de Tucumán, Argentina. 1948. Fuente: Ciudad Universitaria de Tucumán, UNT, San Miguel de Tucumán, 1950, p. 31.

Fig. 04

Eduardo Catalano. Proyecto para estructura en superficie de doble curvatura. Fuente: Gazaneo, J.; Scarone, M., Eduardo Catalano, Instituto de Arte Americano e Investigaciones Estéticas (FADU-UBA), Buenos Aires, 1956, figura 40. 


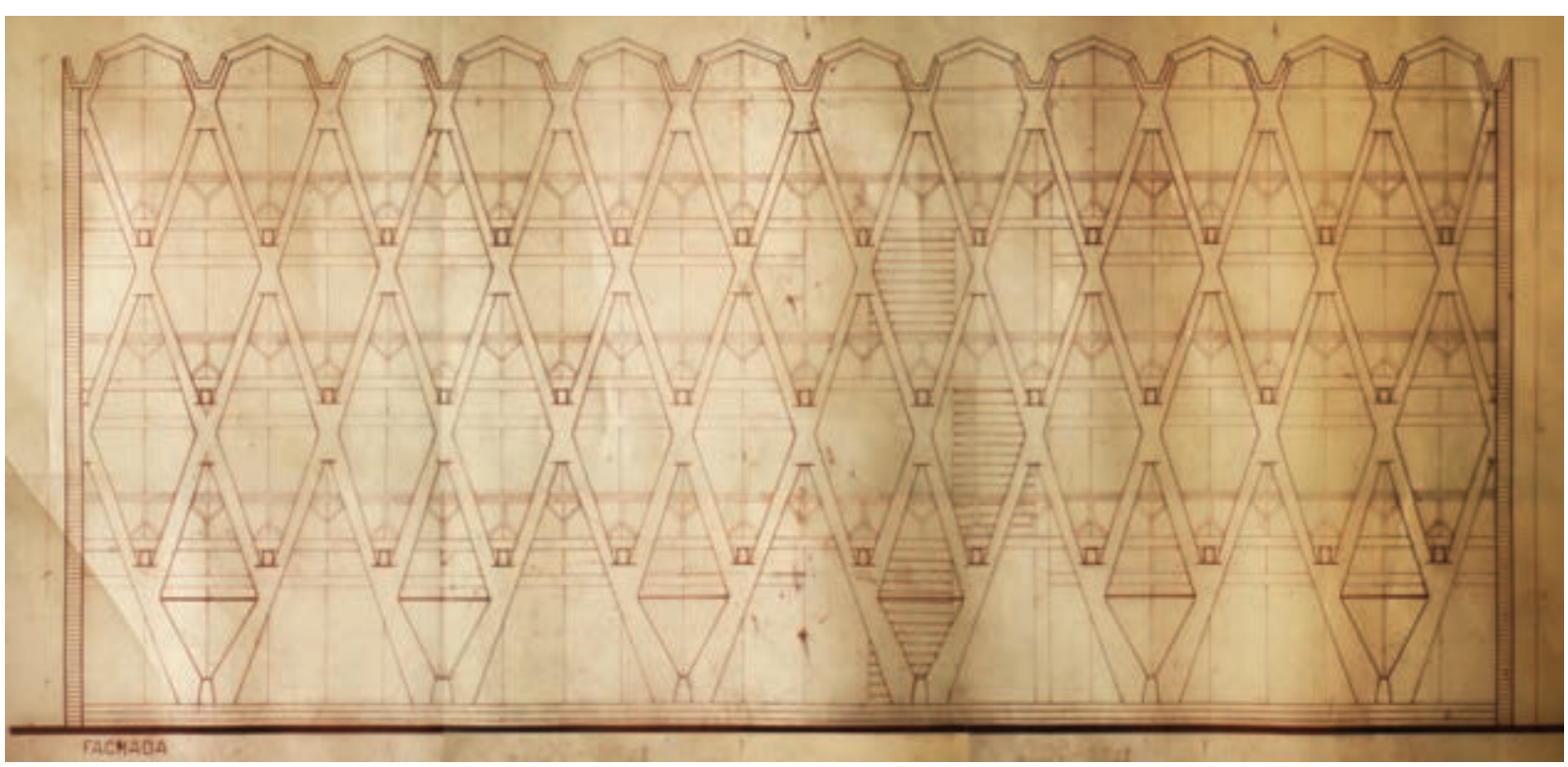

05
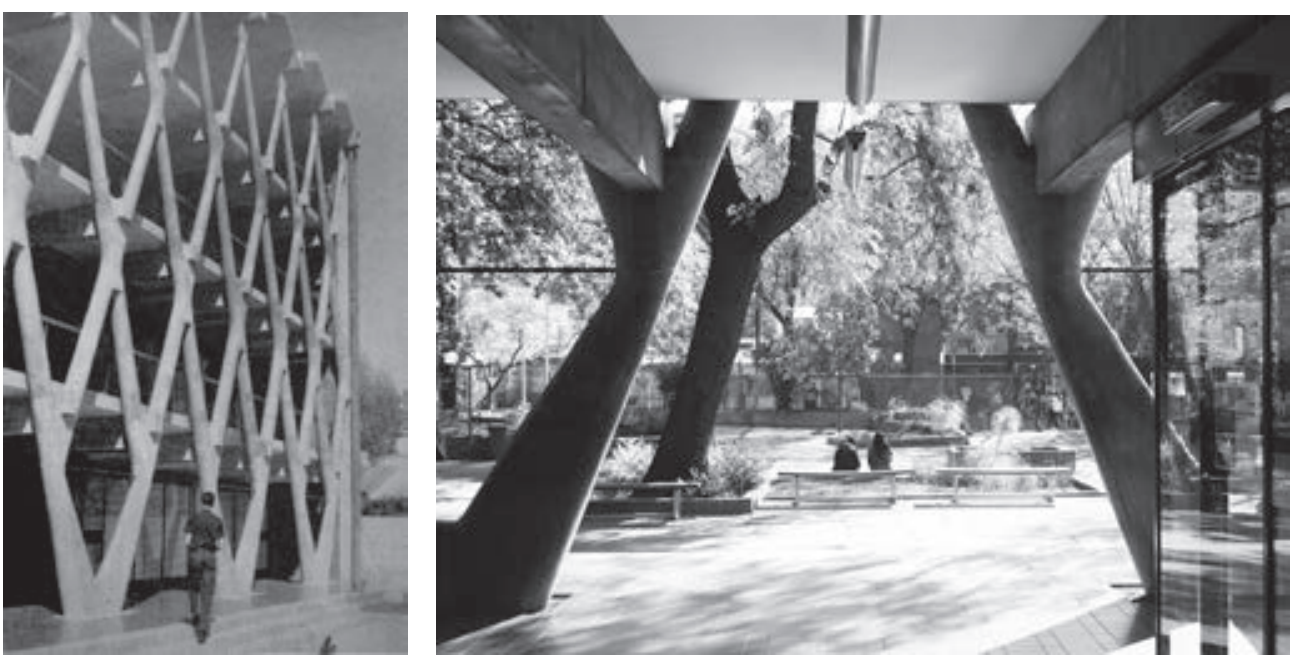

06

07
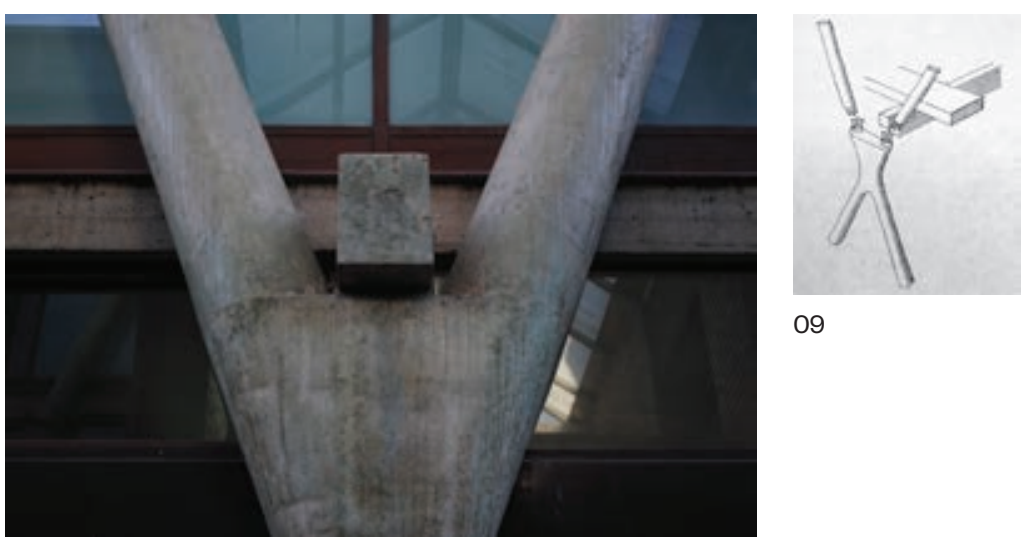

09 
retícula como si se tratara de una gran pieza, cuya inspiración naturalista es deliberada, ya que tanto se puede observar en ella una figuración arbórea como otra antropomórfica (fig. 05). Los estudios de Codina sobre el proyecto revelan que en la composición de esta fachada subyace la utilización del número de oro integrando el ángulo de inclinación de la retícula a un sistema modular de $2,44 \mathrm{~m}$ y dando como resultado intervalos verticales de $3,20 \mathrm{~m}$ entre los niveles superiores de las losas. Un orden matemático que estaría racionalizando ciertas lógicas de la naturaleza, tal como Argan observaba en los procesos proyectuales de Wright (fig. 06). No obstante, al carácter orgánico del proyecto no se debe exclusivamente a los saberes de la geometría sino también a otros factores holísticos y materiales: en primer lugar, cada pieza se integra en un conjunto mayor, donde cada parte está en una posición determinada, materializando un ordenamiento en red, de estructura relacional y no jerárquica entre elementos; la relación entre la estructura y el espacio por medio de la permeabilidad de la trama y de las superficies transparentes por detrás, evita la fachada cortina y simula un diafragma entre el tratamiento del interior y el exterior (fig. 07); el aligeramiento del peso de la masa estructural a medida que asciende, contribuye a ilusión de crecimiento natural; por último, la coloración verdosa del hormigón a la vista intensifica más aún el efecto continuo entre el edificio y el entorno (fig. 08).

El diseño de las articulaciones expone un modo delicado de encastre entre piezas que no da lugar a interpretaciones brutalistas (fig. 09). Como señala Adagio, el edificio se inscribe en un conjunto de obras realizadas en la década del sesenta que acompañan un proceso de alto desarrollo de la industria del hormigón armado en la Argentina y de un interés por la prefabricación, que se combinó con técnicas contructivas artesanales a pie de obra ${ }^{20}$. Sin embargo, a diferencia de otras obras contemporáneas y locales la sistematización de las piezas, el proceso constructivo y el diseño estructural no explican la concepción global del proyecto, que no busca el protagonismo del material. Predominan, en cambio, el juego de múltiples reflejos que se dan a partir de la proyección de sombras de la estructura externa hacia las superficies paralelas de los muros de vidrio retrasados que cierran los talleres y hacia los planos horizontales perpendiculares que materializan las galerías al frente, revestidos en un cemento alisado rojizo. El tipo de concepción organicista de este proyecto gira el foco de atención de la figura a la materia, ya que aquí las ideas formales no se limitan a una analogía naturalista de tipo figurativa sino que se traducen en resultados de gran sensibilidad háptica. Esta orientación se comprende mejor si tenemos en cuenta que en la teoría arquitectónica de Tedeschi la forma arquitectónica es definida por medio de una tríada compuesta por los conceptos de espacio, plástica y escala, donde la componente plástica es la que aporta el "carácter formal de los elementos construidos que limitan el espacio", no sólo a causa de sus propiedades geométricas sino también de cualidades materiales como la textura, la luz y el color, siempre funcionales a una finalidad espacial ${ }^{21}$. En ocasión de explicar las columnas fungiformes proyectadas por Wright en el edificio Johnson, Tedeschi afirmaba:
"[ ] su importancia como hechos técnicos es seguramente inferior a su importancia como formas plásticas determinantes de una situación especial (sic) sumamente nueva y rica. No se trata de una invención que pueda ser aprovechada en cualquier construcción, así como corresponde a una obra
Fig. 05

Enrico Tedeschi. Edificio para la Facultad de Arquitectura de la Universidad de Mendoza, Mendoza, Argentina, 1960-64. Plano de elevación 1:50. Fuente: Archivo Universidad de Mendoza.

Fig. 06

Enrico Tedeschi. Edificio para la Facultad de Arquitectura de la Universidad de Mendoza, Mendoza, Argentina, 1960-64. Fotografía de época.

Fuente: Architectural Review 829, marzo 1966, p. 170.

Fig. 07

Vista desde el interior del vestíbulo de acceso hacia el jardín. Fuente: Fotografía de la autora, 2011.

Fig. 08

Detalle de la unión entre las piezas cónicas, el nudo, la viga transversal y el forjado. Fuente: Fotografía de la autora, 2011.

Fig. 09

Esquema de articulación de los elementos estructurales. Fuente: Summa 85, enero 1975 , p. 73. 


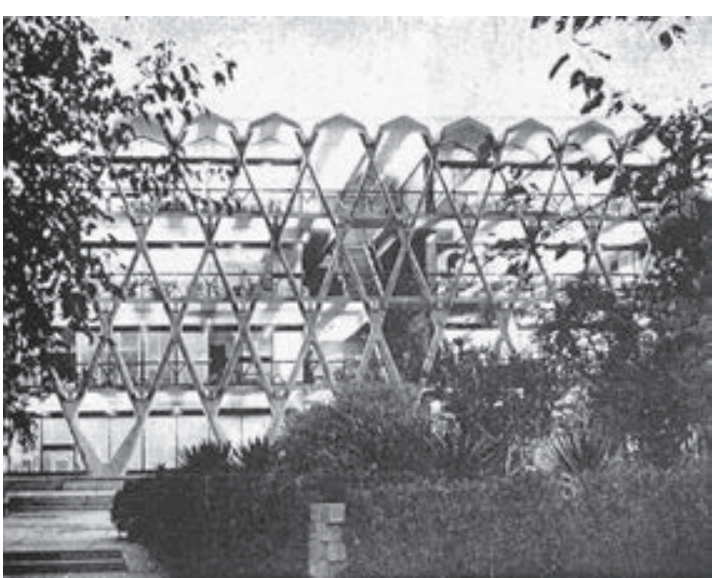

10

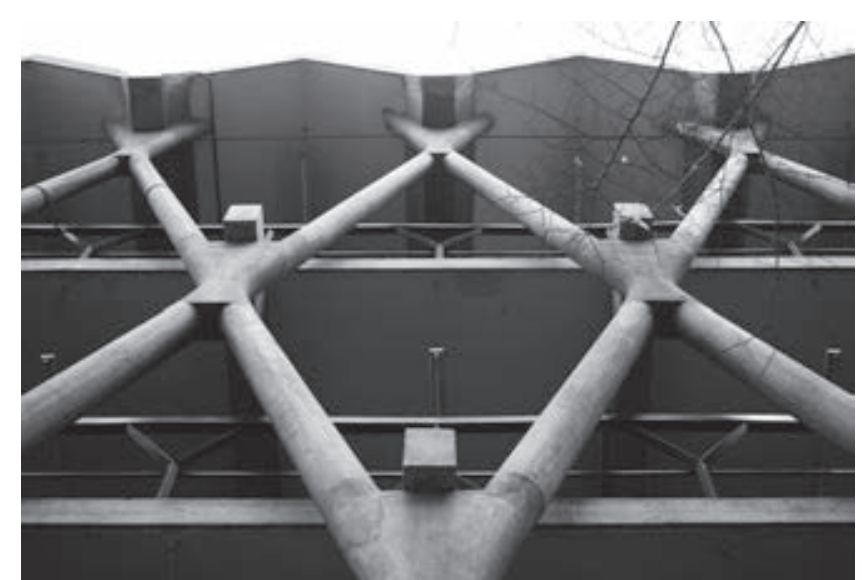

11
Fig. 10

Visión lejana desde el acceso al jardín frontal. La cualidad porosa del edificio se acentúa con el interior iluminado. Fuente: Teoría de la arquitectura, Nueva Visión, Buenos Aires, 1969, $2^{\circ}$ edición, figura 40.

Fig. 11

Vista de la estructura hacia la unión con la cubierta. Fuente: Fotografía de la autora, 2011. de verdadero carácter técnico, sino de una pieza única, creada con gran maestría constructiva para una finalidad espacial y para un único edificio"22

Las piezas singulares del edificio para la Facultad de Mendoza son los nudos y los pies de apoyo en "V", pensados y construidos a pie de obra exclusivamente para este proyecto. La plasticidad y liviandad de los elementos de la estructura habilitan un lenguaje híbrido entre tectónico y textil que da cuenta de una preocupación por la imbricación profunda entre forma, materia y técnica sin renunciar a una necesaria dosis de abstracción propia de los principios de la arquitectura moderna más ortodoxa, implacable en la determinación rotunda de su volumetría y en la flexibilidad funcional de su planta. Sin embargo, la apariencia objetual del edificio es evadida; a cierta distancia, su fachada se percibe como un fragmento del paisaje, y su extensión a lo ancho, según el punto de vista del observador es más o menos reconocible (fig. 10). En parte, ello se debe a que nunca se aprecia el volumen en sus cuatro caras, ya que dos de ellas son muros medianeros y, al estar revestidos en ladrillo cerámico, enfatizan la interrupción de la continuidad de la retícula a ambos lados. En la dirección vertical, en cambio, el encuentro con la cubierta se resuelve de un modo más suave; a pesar de que la retícula se encuentra retrasada, a cierta distancia gana la continuidad entre las losetas y los postes inclinados, debido a que la delgadez y el plegado de las mismas cierra en forma cóncava sus extremos (fig. 11).

El proyecto se introdujo positivamente en la valoración crítica de la historia de la arquitectura moderna en Argentina por dialogar con temas vigentes en la práctica profesional en el país. Sin embargo, anticipaba conceptualmente no una idea, sino un conjunto de ideas sobre la materia muy actuales en la disciplina; opera del modo en el que -observa Stan Allen- lo hacen algunas tendencias arquitectónicas de metáfora geológica en el siglo XXI: son ensamblajes abiertos y envolventes porosas cuya forma icónica o contorno definido no es tan importante como la relación que establecen de parte a parte y entre interior y exterior ${ }^{23}$. Una concepción paisajística de la arquitectura que, con otras características, el mismo Tedeschi había encontrado en sus viajes por el Cuzco en la cultura arquitectónica barroca de América Latina ${ }^{24}$. RA 


\section{Notas}

01. WAENERBERG, A., "Organic: A brief history of the concept", en Structurist, 2007-2008, 47/48, pp. 41-47.

02. HVATTUM, M., "«Unfolding from within». Modern architecture and the dream of organic totality", en The Journal of Architecture, 2006, 11 (4), p. 497.

03. ZEVI, B., Storia dell 'architettura moderna, Einaudi, Turín, 1950.

04. SAMONÀ, G., "Sull' architettura di Frank Lloyd Wright", en Metron, 1951, 41-42, p. 41.

05. ARGAN, G. C., "Introduzione a Wright", en Metron, 1947, 18, p. 10.

06. POPE, A., "Sobre la mímesis moderna de la naturaleza. Redes, herramientas y planteamientos ecológicos", en Ra, Revista de arquitectura, 2018, 20, p. 248.

07. TEDESCHI, E., "Arquitectura orgánica", en Nuestra Arquitectura, 1952, 273, p. 123.

08. TEDESCHI, E., Frank Lloyd Wright, Nueva Visión, Buenos Aires, 1955, p. 31.

09. MOHOLY-NAGY, L., Vision in Motion, Paul Theobald, Chicago, 1947.

10. TEDESCHI, E., "Prefabricación en Inglaterra: Nursery en Garston", en Nuestra Arquitectura, 1952, 279, pp. 278-290.

11. TEDESCHI, E., "Estructura contemporánea en arquitectura", en Nuestra Arquitectura, 1953, 286, pp. 152-154.

12. SEVERUD, F., "La natura è una fonte di ispirazione strutturale", en Metron, 1945, 4-5, pp. 21-29.

13. TEDESCHI, E., Teoría de la arquitectura. Nueva Visión, Buenos Aires, 1963.

14. TEDESCHI, Enrico, "La arquitectura contemporánea frente al progreso de las ciencias y de las técnicas", en Revista de la Universidad Nacional de Córdoba, diciembre 1968, p. 588.

15. PIZZETTI, J., "Los nuevos mundos de la arquitectura estructural”, en Nueva Visión, 1951,1, p. 13

16. El proceso proyectual y constructivo de esta obra fue estudiado en profundidad y por primera vez en la tesis de maestría de Leonardo García Codina. Véase: CODINA, Leonardo, La estructura como instrumento de una idea. Enrico Tedeschi y el proyecto de la Facultad de Arquitectura de la Universidad de Mendoza. Pontificia Universidad Católica de Chile, 2004. Publicada por Ediciones 1:100, Buenos Aires, 2013.

17. CODINA, Leonardo, La estructura como instrumento de una idea. Enrico Tedeschi . El proyecto de la Facultad de Arquitectura de la Universidad de Mendoza. Buenos Aires, Ediciones 1:100, 2013.

18. La empresa italiana SCAC (Società Cementi Armati Centrifugati) fue fundada en Trentino, Italia, en 1920 y se dedicaba a la fabricación de elementos de hormigón para obras de arquitectura y construcción civil. En la década del cincuenta instaló varios establecimientos industriales en Argentina. Véase: GIOVANNARDI, F., Enrico Tedeschi dal sogno alla tragedia argentina, Pàtron, Bologna, 2016.

19. TEDESCHI, E., "La arquitectura contemporánea frente al progreso de las ciencias y de las técnicas", en Revista de la Universidad Nacional de Córdoba, diciembre 1968, p. 612.

20. ADAGIO, Noemí, "Forma y tecnología. El edificio de la Facultad de Arquitectura en Mendoza (1962-1964)", A.A.V.V., en Enrico Tedeschi work in progress, EDIUM, Mendoza, 2013, pp-115-122.

21. TEDESCHI, E., Teoría de la arquitectura, Nueva Visión, Buenos Aires, 1969, $2^{a}$ edición, p. 209.

22. TEDESCHI, E., "La arquitectura contemporánea frente al progreso de las ciencias y de las técnicas", en Revista de la Universidad Nacional de Córdoba diciembre 1968, p. 605.

23. ALLEN, S. "Geological form. Towards a vital materialism in Architecture", Wilke, B. (ed.), en Four projects, Ohio State University, Columbus, 2017, p. 15.

24. Respecto de la sensibilidad ambiental de Tedeschi en su mirada a la arquitectura barroca del Perú, véase: ALVITE, Silvia, "Enrico Tedeschi y la crítica fotográfica en el paisaje arquitectónico latinoamericano", Anales del IAA, 45, pp. 73-86.
Referencias Bibliográficas

- ADAGIO, N.; SELLA, A. (eds.), Enrico Tedeschi: Work in Progress, EDIUM, Mendoza, 2013

- ALVITE, Silvia, Espacio, región, paisaje, organicismo e historia en el itinerario de Enrico Tedeschi. Arquitectura y urbanismo en Argentina (1948-1978), Tesis de doctorado, Universidad de Buenos Aires, Facultad de Arquitectura, Diseño y Urbanismo, 2019.

- BULLRICH, Francisco, Arquitectura Argentina Contemporánea, Nueva Visión, Buenos Aires, 1963

- DE GRAZIA, Francisco, "Tedeschi, el espacialista confeso", en Tedeschi, E. Una introducción a la historia de la arquitectura, Reverté, Barcelona, 2017

- DESCOTTE, Mario Luis, Historia de la Universidad de Mendoza (1960-2010), EDIUM, Mendoza, 2010.

- LIERNUR, Jorge Francisco, Arquitectura en la Argentina del siglo XX: la construcción de la modernidad, Fondo Nacional de la Artes, Buenos Aires, 2001.

- MARINO, Fabio, Enrico Tedeschi. 19101978. Dall'Italia all 'Argentina, Tesis de Maestría, Politecnico di Milano, 2014.

- TEDESCHI, Enrico, "Escuela universitaria de arquitectura", en Nuestra Arquitectura, 433, 1966, pp. 34-36.

- TEDESCHI, Enrico, "Un aporte a las construcciones antisísmicas", en Summa, 85,1975, p. 73.
RA. Revista de Arquitectura Núm. 22 - 2020 P. $160-169$ 